\title{
On the Research of Adaptive Frequency Hopping in the Wireless Communication
}

\author{
LIANG Xiao-hu \\ Institute of Communication Engineering \\ PLAUST \\ Nanjing, China \\ E-mail: liangxiaohu688@163.com
}

\author{
CHENG Lei \\ Institute of Communication Engineering \\ PLAUST \\ Nanjing, China \\ E-mail: chenglei@163.com
}

\begin{abstract}
The multipath fading and interference problems in the wireless communication lead to the link quality decrease severely. However, the conventional frequency hopping scheme cannot solve the problems in an effective way. Adaptive frequency hopping scheme, which is based on the automatically link quality analysis, can avoid the 'bad' frequencies and guarantee the desired level of channel quality. The system is analyzed based on the adaptive frequency hopping model equipped with link quality analyzer which is different from the traditional one. In the paper, some newly proposed adaptive frequency hopping schemes have been summarized, which provide an important reference for the practical application.
\end{abstract}

Keywords-adaptive frequency-hopping; frequency adaptive algorithm; frequency diversity

\section{INTRODUCTION}

Adaptive frequency hopping is a hot issue in the wireless communication in recent years. The channel quality analysis, frequency adaptive, power adaptive, adaptive modulation and adaptive coding are the aspects of adaptive frequency hopping system. The frequency adaptive is one of the most important parts in the adaptive frequency hopping system.

In the civil application, as for sharing the same range of ISM (Industry, Science and Medical) frequency, the users of microwave oven, wireless telephone and the wireless personal area networks (WPAN ${ }^{11]}$ ) always interfere each other severely. Adaptive frequency hopping techniques have been adopted in Bluetooth ${ }^{[2]}$ communication to reduce the fluctuation of the wireless fading channel and the interference of the nearby users in the communication. In the military application, the anti-jamming ${ }^{[3]}$ ability of the satellite communication and the high frequency communication is an important factor to be considered in the practical application. The detective probability for the nontarget users must be low as possible, while the intercepted probability for the hostile users also should be low in the wireless communication. With development of the modern information technique, the conventional adaptive frequency hopping cannot satisfy the increasingly need of the future electronic information warfare. Comparing with the traditional frequency hopping scheme, adaptive frequency hopping techniques have the advantages of the better flexibility, the better reliability and the better security. In the wireless communication, adaptive frequency hopping system can automatically modify the modulation, coding rate and the sending power, reducing the external interference and enemy jamming in the channel. Then the adaptive frequency hopping systems guarantee the communication quality as possible.

\section{ADAPTIVE FREQUENCY PRINCIPLE}

In the [4], J.Zander proposed the adaptive frequency hopping for the first time. As shown in the Figure 1, adaptive frequency hopping communication has three steps: communication setup, frequency sweeping and communication holding. In the first step, based on the clock synchronization and frame synchronization, goal of adaptive frequency hopping is to make the hopset synchronize between the sender and receiver. In the second step, the link quality analyzer detects all the members of hopset to determine which channels have been interfered according to adaptive frequency hopping scheme. In the communication keeping, the link quality analyzer will inform the sender of the changed channel condition which caused by interference.

Adaptive frequency hopping system has not only the anti-jamming of the conventional frequency hopping, but also the function of frequency adaptive and power adaptive. Based on the automatically link quality analysis, adaptive frequency hopping system can remove the bad channels from the hopset timely and gain the desired level of frequency diversity. Thus, adaptive frequency hopping systems have the ability of reducing single-tone interference, multiple-tone interference, and other kinds of jamming in the channel.

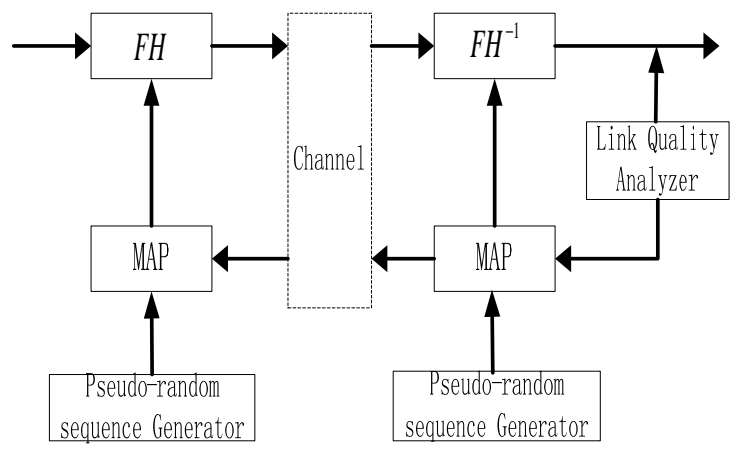

Figure 1. Adaptive Frequency-Hopping Model

\section{ANALYSIS OF ADAPTIVE FREQUENCY HOPPING SCHEME}

\section{A. Conventional Adaptive Frequency Hopping}

In order to analyze the adaptive frequency-hopping system, the reference [4] proposed a simple two-state 
channel model in the wireless communication, which used the Markov Chain to simulate the transition of the good state and the bad state.

The Gilbert-Elliot model is shown in the Figure 2. $r$ is the probability of leaving the good state, while $S$ is the probability of leaving the bad state. In the model, every channel has been assigned with two states: the bad state and the good state. When the bit error rate (BER) of the channel is above the threshold, the channel will be attached to the bad state. In the same way, the channel will be attached to the good state in the low BER. The approach to judge the state of channel is relying on the power of the external interference, intensity of the received signal and BER.

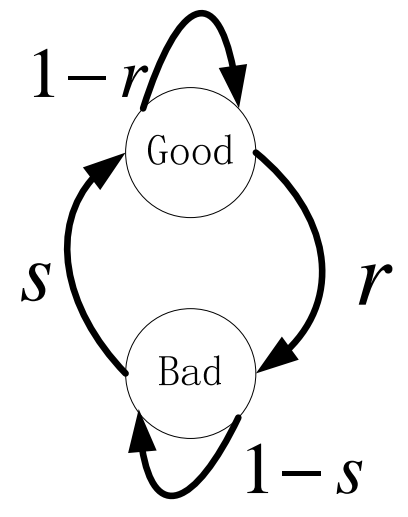

Figure 2. Two-State Gilbert-Elliot Model.

\section{B. Utility Based Adaptive Frequency Hopping(UBAFH)}

The purpose of conventional adaptive frequency hopping scheme is to identify bad channels which are eventually excluded from the hopset. By assigning different usage probabilities to the channel based on the conditions of channel, UBAFH ${ }^{[5]}$ can make full use of all the available frequencies comparing with the traditional adaptive frequency-hopping. The advantage of UBAFH is to achieve a high level of frequency diversity with the upper and lower limits of the usage probability. Besides, UBAFH can avoid the hopset centralizing only in the channels with good condition. In this way, UBAFH guarantees the ability to withstand the external interference and the frequency selective fading in the wireless multipath channel.

In UBAFH, the estimated packet error rates (PERs) are firstly mapped to a probability density function $f:[0,1] \rightarrow\left[P_{M I N}, P_{M A X}\right]$, defining usage probabilities for each channel. The higher usage probabilities will be assigned to the channels which have lower PER. The setting of $P_{\text {MIN }}$ is to guarantee that the bad channels have the probability to be used. The $P_{\text {MAX }}$ is to gain the desired level of frequency diversity, avoiding that there are only some good channels being used. The probability density function is composed of three functions which can be resolved as $f=f_{1} \circ f_{2} \circ f_{3}$.

$$
f_{1}: \operatorname{PER}\left(c_{i}\right) \rightarrow f_{1}\left(\operatorname{PER}\left(c_{i}\right)\right)=\left(1-\operatorname{PER}\left(c_{i}\right)\right)^{\alpha} / \sum_{j=1}^{M}\left(1-\operatorname{PER}\left(c_{j}\right)\right)^{\alpha}
$$

$\sum_{i=1}^{M} f_{1}\left(\operatorname{PER}\left(c_{i}\right)\right)=1, \quad \alpha$ is used to change the usage probability of channels. While higher values of $\alpha$ lead to scenarios where only the best frequencies are actually used, the lower values of $\alpha$ can make the channel usage probabilities equal.

$f_{2}$ increases the values obtained using $f_{1}$ by adding $P_{M I N}$. In order to gain a new probability density function, we scale those values by a factor of $1-M \cdot P_{M I N}$. In the end, the function $f_{3}$ truncates channel usage probabilities greater than $P_{M A X}$ and reallocates the greater parts to the all other channels. At this time, the progress of mapping has finished.

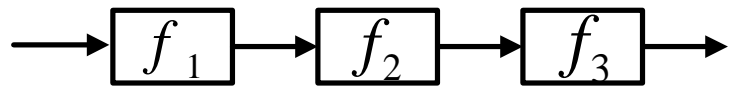

Figure 3. Mapping scheme

\section{Robust Adaptive Frequency Hopping(RAFH)}


Unlike the traditional adaptive frequency hopping scheme which identities channels good or bad by thresholdbased on-off decision, $\mathrm{RAFH}^{[6]}$ instead allocated each

$$
\begin{aligned}
& \text { //Initialize } \\
& \mathrm{p} \leftarrow[1 / M \ldots 1 / M] \\
& \text { //PER estimation } \\
& \text { Reset timer } \mathrm{t} \leftarrow \mathrm{T} \\
& n_{t}=\left(n_{1, t}, \ldots, n_{M, t}\right) \leftarrow 0 \\
& n_{e}=\left(n_{1, e}, \ldots, n_{M, e}\right) \leftarrow 0 \\
& \text { While (True) do } \\
& \text { If current transmission uses frequency } i \text { then } \\
& n_{i, t} \leftarrow n_{i, t}+1 \\
& \text { End if } \\
& \text { If Transmission fails then } \\
& n_{e, t} \leftarrow n_{e, t}+1 \\
& \text { End if } \\
& a_{i}=P E R_{i} \leftarrow n_{i, e} / n_{i, t} \\
& p \leftarrow \arg \min _{p} \sum_{i=1}^{M} p_{i} \log p_{i} \text { Such that } \\
& \sum_{i=1}^{M} a_{i} p_{i} \leq \xi \text { and } \sum_{i=1}^{M} p_{i}=1 \\
& p_{i}=1 / K \text { For the top } K \text { channels and } p_{i}=0
\end{aligned}
$$

channel a different hop probability as a decreasing function of the packet error rate. RAFH can not only overcome the disadvantages of the conventional adaptive frequencyhopping, but also can gain the desired level frequency diversity. On the one hand, RAFH can reduce the fluctuation over time under the dynamic jamming. On the other hand, RAFH has a higher complexity which needs more computation in the processing. RAFH is given in the following. The PER of every channel will be measured in every interval of $\mathrm{T}$. It will begin to update the hopping probability $\mathrm{p}$ when the total PER exceed the pre-assigned threshold. By allocating the hop probability considering the magnitude of the measured PER, RAFH can reflect the channel condition more accurately than the conventional adaptive frequency-hopping. The hop probability will be assigned with the total PER under the threshold. However, it is not enough to provide robustness against the random interference. Because of the random arrival of interference source, the measured PER cannot contribute to the nearly arriving interference. So it is important to make the hop probability random as possible, then we can gain more frequency diversity to combat for random interference.

\section{Smooth Adaptive Frequency Hopping(SAFH)}

In the [7], the paper proposed a kind cross-layer scheme of smooth adaptive frequency hopping, which is based on exponential smoothing filter by assigning usage probabilities to all channels. In this way, SAFH can estimate and predict the channel conditions. Moreover, the upper layers are available to adapt SAFH by changing the parameters in a cross-layer approach. According to simulation, SAFH outperforms conventional frequency hopping and AFH. In the same time, comparing with the UBAFH and RAFH, SAFH has more advantages in the aspects of frequency diversity and provides with more robust ability for combating the changing channel.

As is shown in the Figure 4, SAFH has four steps: channel classification, channel prediction, mapping and hopset generation. While RAFH and UAFH have only three steps: channel classification, mapping and hopset generation; AFH has only channel classification and hopset generation.

In the first step, by analyzing the channel quality and measuring the frame error rate, SAFH can determine the frequencies and the power of interference at the present. SAFH can minimize the risk of incorrect classification 。

In the second step, SAFH uses exponential smoothing to predict the frame error rate $\left(F E R_{i}^{\prime}, \forall i \in\{1 . . N\}\right)$. Equation 1 is the smoothing filter which can calculates the frame error rate by the last measurement. $\alpha$ is a factor between 0 and 1 . The filter will give more weight to recent data and reducing smoothing effect when $\alpha$ approaching 1 .

$$
\left\{\begin{array}{l}
F E R_{i}^{\prime}(t+1)=\alpha^{*} F E R_{i}(t)+(1-\alpha)^{*} F E R_{i}^{\prime}(t) \\
F E R_{i}^{\prime}(1)=F E R_{i}(0)
\end{array}\right.
$$

In the third step, we map the $F E R_{i}^{\prime}(t+1)$ to a discrete probability distribution to satisfy the desired level of the channel quality. Comparing with conventional adaptive frequency hopping avoiding a bad channel entirely, SAFH assigns a probability which is a function of its distance from the threshold.

In the forth step, SAFH makes the probability distribution map to a hopset. 


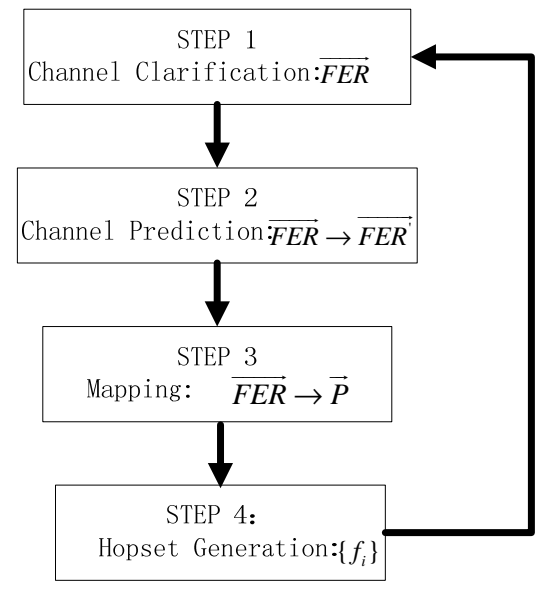

Figure 4. Block Diagram of the SAFH

\section{E. Enhanced Adaptive Frequency Hopping(EAFH)}

In order to exclude the bad channels being interfered from the hopset effectively, the paper [8] presented an EAFH scheme based on the feeding back mechanism of PER. By monitoring the packet error bit of the channels, EAFH can determine the right number of channels to be removed from the hopset. Then EAFH will decide which channel to be removed according to the packet error rate of individual channel. The channels are parted into three groups. The group of $\mathrm{A}$ is used to transmit multiple-slot packets, while B group using single-slot packet, and C be the group of excluded channels. As for the channel condition is time-varying, the channel number of each group may change every time. EAFH can balance the usage rate of each channel and the average usage rate of all channels, because EAFH can modify the numbers of three channels based on the PER of every channel.

Every user monitors the packet error rate of each channel, and detects all other channels' packet error rate at the same time. By determine the channel which group should be put into, EAFH can mitigate frequency dynamic interference and frequency static interference.

The way to eliminate the frequency dynamic interference is that EAFH begin to adjust the channels in the hopset and the length of the packet, when users detect the external interference. In the initialization, the $\mathrm{N}$ channels are assigned to the A group, the $2 \mathrm{~N}$ channels are parted into the group $\mathrm{C}$ and the rest of channels belong to the group $\mathrm{B}$. After initialization, EAFH will keep an eye on the packet error rate all the time. While the packet error rate of channel in group A appears higher than the average packet error rate of all channels, EAFH will switch it with a channel which has lower PER in group B or C. In the same way, switching between group $\mathrm{B}$ and $\mathrm{C}$ may occur in certain conditions.
The swap of the three groups can eliminate the unbalance of initial assignment and periodically updates the channels of each group.

While users detect the severe interference in a certain channel for long time, i.e. PER>0.5, EAFH will recognize that it is the frequency interference in the channels and transitorily excluded the interfered channels from the hopset for a duration of $\mathrm{T}$. These channels belong to the subgroup of $\mathrm{C}$, and channels in this subgroup will not join the switching progress.

\section{CONCLUSIONS}

The paper makes a comparison of the four newly proposed adaptive frequency hopping schemes in the wireless communication. Comparing with the conventional adaptive frequency hopping, the new adaptive frequency hopping schemes instead map the packet error rate to the usage probability density function, which can guarantee the desired level of frequency diversity. The new adaptive frequency hopping schemes are robust in interference antijamming. Besides, those newly proposed adaptive frequency hopping schemes can be suitable for different range of frequency, such as HF (High Frequency), UHF (Ultra High Frequency) and SHF (Super High Frequency). The unsympathetic between uplink and downlink should be considered in the practical engineering application.

\section{REFERENCES}

[1] Ralph Tanbourgi, Jens P. Elsner, Holger Jakel, Friedrich K.Jondral, "Adaptive Frequency Hopping in Ad Hoc Networks with Rayleigh Fading and Imperfect Sensing”, IEEE Wireless Communications Letters, vol.1, No.3, June 2012.

[2] Seung-Hwan Lee, Yong-Hwan Lee, “Adaptive Frequency Hopping for Bluetooth Robust to WLAN Interference”, IEEE Communications Letters, vol.13, No.9, September 2009.

[3] Reza Nikjah, Norman C. Beaulieu, “On Antijamming in General CDMA Systems-Part II : Antijamming Performance of Coded Multicarrier Frequency-Hopping Spread Spectrum Systems”, IEEE transactions on wireless communications, vol.7, No.3, March 2008.

[4] J. Zander, G. Malmgren, “Adaptive frequency hopping in HF communications”, IEE Proc. Comm., vol. 142, No. 2, April 1995,pp. 99105, 1995.

[5] Luca Stabellini, Lei Shi, Ahmad Al Rifai, Juan Espino, Veatriki Magoula, "A New Probabilistic Approach for Adaptive Frequency Hopping”, 2009.

[6] K.-J. Park, T.R. Park, C.D. Schmitz, L. Sha, "Design of robust adaptive frequency hopping for wireless medical telemetry systems”, IET Commun., vol. 4, lss.2, pp.178-191,2010.

[7] Sami Ben Cheikh, Tim Esemann, Horst Hellbruck, "SAFH-Smooth Adaptive Frequency Hopping”, 2011.

[8] Alex Chia-Chun Hsu, David S.L. Wei, C.-C. Jay Kuo, Norio Shiratori, Chung-Ju Chang, "Enhanced Adaptive Frequency Hopping for Wireless Personal Area Networks in a Coexistence Environment”, IEEE GLOBECOM proceedings,2009. 